\title{
A GLC CONTROLLER FOR THE CONTROL OF THE METHYL METHACRYLATE POLYMERIZATION
}

\author{
Mehdi Rafizadeh ${ }^{1}$, Ahmad Afshar ${ }^{2}$, and Hassan Gharghi ${ }^{2}$ \\ ${ }^{1}$ Polymer Engineering Department \\ ${ }^{2}$ Electrical Engineering Department \\ AmirKabir University of Technology \\ Tehran, IRAN, P.O.Box 15875-4413 \\ Email:mehdi@cic.aku.ac.ir
}

\begin{abstract}
To produce a certain polymer with desired properties, some of polymerization variables should be controlled. In this paper, a oil bath was designed and constructed for the heating and cooling of the reactor. Then, a mechanistic model was developed for the solution polymerization of methyl methacrylate in a batch reactor. This model is highly non-linear. A Globally Linearizing Control (GLC) strategy was applied to control the reactor temperature. A linear PI controller was designed to eliminate the error. The controller performance was viable and the previously developed GLC method is suitable for the oil heating system. Copyright (C) 2002 IFAC
\end{abstract}

Keywords: Chemical industry, Feedback control, Linear control systems, Linearization, Modeling.

\section{INTRODUCTION}

Polymer properties normally are determined during the polymerization process. The importance of reactor effective control has been considered in recent decades. Due to the cage, gel, and glass effects, kinetic of polymerization is complex. Therefore, the process is highly non-linear and time variant. Hence, it is a challenging task to control polymerization reactor. Although, the best approach is measuring the final product properties, but there are a few appropriate measuring elements to do so. Alternative approach is to control some critical variables, such as reactor temperature.

Batch reactor is preferable to produce a small amount of polymers and copolymers. However, the batch

\footnotetext{
${ }^{1}$ To whom all correspondence should be address
}

reactor is naturally dynamic and its dynamic features are time variant even in a wide range, during the course of polymerization. A reliable dynamic model is necessary to have an effective controller performance. Polymerization modeling has been witnessed a considerable increase in the understanding of various aspects of the complex polymerization systems.

Louie et al (1985) reviewed gel effect models and their theoretical foundations. Then, they extended a previously developed bulk polymerization model for the solution polymerization of methyl methacrylate.

Usually, researchers have preferred to carry out control studies based on the experimental model of the polymerization. However, it is more preferable to relate controller parameters to the process parameters and variables. Tzouanas and Shah (1989) suggested an adaptive pole displacement control approach were 
used for the batch polymerization control. They approximated the monomer conversion using on-line measurement of reactive mixture density. They, then, used ARMA method to estimate the transfer function parameters. Mendoza-Bustos et al (1990) used a computerized pilot plant for undergraduate control laboratory. They derived a first order plus dead time transfer function for polymerization. The time constant and dead time were estimated by open loop dynamic experiments. Then, they designed different controllers. Peterson et al (1989) presented a nonlinear predictive strategy for semi-batch polymerization of MMA. They used a mechanistic model consisting eight states. Although, they didn't conduct any experiments, but their simulation results were satisfactory. Penlidis et al (1992) reviewed a mechanistic model for bulk and solution free radical polymerization for control purposes and examined five models with different level of complexities. Soroush and Kravaris (1992) applied a Global Linearizing Control (GLC) to control reactor temperature. They used a mechanistic model and compared the performance of a conventional PID and the GLC controllers. The result for tracking an optimum temperature trajectory was satisfactory. Clarke-Pringle and MacGregor (1997) studied the temperature control of a semi-batch industrial reactor. They suggested a non-linear strategy coupled with a extended Kalman filter. They applied energy balances for the reactor and jacket and estimated process parameters. They didn't mention their monomer and polymer. Mutha et al (1997) suggested a non-linear model based controller and performed their experiments on solution polymerization of MMA in a small reactor. They used a new estimator as well as Kalman filter. Wright and Kravaris (1997) used a gray box model for polymerization and designed a non-linear controller. They assumed an exponential form for the conversion of the monomer and estimated the heat generation by an experimental model.

Berber et al (1998) presented a six states model and one step Newton method and designed a simple nonlinear controller for the temperature control.

This paper presents the application of the GLC method for a batch solution polymerization reactor. An external linear PI controller is coupled with the GLC structure. The controller is applied to control the reactor temperature of solution polymerization of MMA. Experimental study shows that controller performance is very good.

\section{POLYMERIZATION MECHANISM}

Methyl methacrylate is normally produced by a free radical, chain addition polymerization. Free radical polymerization consists of three main steps: initiation, propagation, and termination. Free radicals are formed via decomposition of the initiator. Once formed, these radicals propagate by reacting with surrounding monomers to produce long polymer chains; the active site being shifted to the end of the chain when a new monomer is added. During the propagation, millions of monomers are added to $P_{1}^{o}$ radicals. During termination, due to reactions among free radicals, the concentration of radicals decreases. Termination is conducted by combination or disproportionate reactions. At a time, there are minor quantities of radicals; the propagation step takes place more than the termination step. With chain transfer reactions to other species, the active free radicals are converted to dead polymer. Table 1 gives the basic free radical polymerization mechanism.

Table 1. Mechanism of free radical polymerization

\begin{tabular}{|c|c|}
\hline Initiation & 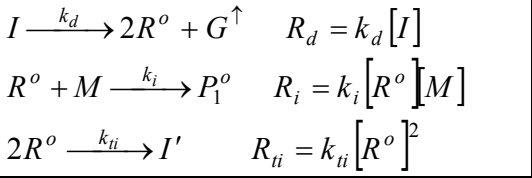 \\
\hline Propagation & $P_{n}^{o}+M \stackrel{k_{p}}{\longrightarrow} P_{n+1}^{o} R_{p}=k_{p}[M]\left[P_{n}^{o}\right]$ \\
\hline Termination & $\begin{array}{l}P_{n}^{o}+P_{m}^{o} \stackrel{k_{t c}}{\longrightarrow} D_{n+m} \quad R_{t c}=k_{t c}\left[P_{n}^{o}\left\lceil P_{m}^{o}\right]\right. \\
P_{n}^{o}+P_{m}^{o} \stackrel{k_{t d}}{\longrightarrow} D_{n}+D_{m} R_{t d}=k_{t d}\left[P_{n}^{o}\left[P_{n}\right.\right.\end{array}$ \\
\hline Transfer & $\begin{array}{l}P_{n}^{o}+M \stackrel{k_{f}}{\longrightarrow} P_{1}^{o}+D_{n} R_{f}=k_{f}[M]\left[P_{n}^{o}\right] \\
P_{n}^{o}+M \stackrel{k_{s}}{\longrightarrow} s^{o}+D_{n} R_{s}=k_{s}[M]\left[P_{n}^{o}\right]\end{array}$ \\
\hline
\end{tabular}

The free radical polymerization rate decreases due to consumption of the monomer and initiator concentration. However, due to viscosity increase beyond a certain conversion there is a sudden increase in the polymerization rate. This effect is called Trommsdorff or gel effect.

\section{EXPERIMENTAL SETUP}

A schematic representation of the experimental batch reactor setup is shown in Figure 1. The reactor is a one-liter Bucchi type jacketed, cylindrical glass vessel. The mixture is agitated by a multipaddle agitator. The speed of the stirrer is kept constant throughout the course of polymerization. 


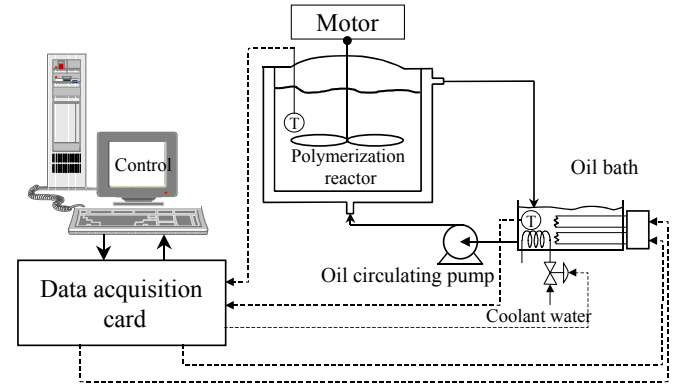

Figure 1. Schematic of experimental system.

A Pentium II $500 \mathrm{~Hz}$ computer is connected to the reactor via an ADCPWM-01 analog/digital I/O data acquisition card. The analog signals of the measuring elements are converted to voltage and amplified to 0 5 volts. The data acquisition software is developed internally.

The necessary heating or cooling of the reactant mixture is continuously supplied through the jacket of the reactor. The heating oil is circulated by a gear pump. The pump flow rate is $15 \mathrm{lit} / \mathrm{min}$. The heating/cooling system of the oil consists of two $1500 \mathrm{w}$ electrical heaters and one coolant water coil which is operated by a On/Off Acco brand solenoid valve. Two Resistance Temperature Detectors (RTD), are used with accuracy of $\pm 0.2{ }^{\circ} \mathrm{C}$.

\section{MATHEMATICAL MODELING}

In a batch reactor, all reactants are added initially. Quasi Steady State Approximation (QSSA) is used to simplify control studies. Number of equations can be reduced to finite numbers by moments. Definitions of moments are (Louie et al (1985)):

$\lambda_{k}=\sum_{n=1}^{\infty} n^{k}\left[P_{n}^{\circ}\right], \quad \mu_{k}=\sum_{n=1}^{\infty} n^{k}\left[D_{n}\right]$

Which $\lambda_{k}$ and $\mu_{k}$ are alive and dead polymer chain $k^{\text {th }}$ moments, respectively. Table 2 shows the governing equations. The polymer production in this reaction is accomplished with reduction in the mixture volume. The instantaneous volume of reactant mixture is:

$V=\frac{M_{0}}{\rho_{m}}(1-\varepsilon x+\beta)$

where $x$ is the conversion, $\varepsilon$ is the volume reduction factor and $\beta$ is defined as: $\beta=\frac{f_{s}}{1-f_{s}}$

where $f_{s}$ is the initial volume fraction of the solvent.

Monomer concentration is given by:

$x=\frac{[M]_{0} V_{0}-[M] V}{[M]_{0} V_{0}}$

Table 2. Governing equations using the QSSA approximation

$$
\begin{aligned}
& \frac{d x}{d t}=\frac{2 f k_{d}}{[M]_{0} V_{0}}[I]+\left(k_{p}+k_{f}\right)(1-x) \lambda_{0} \\
& \frac{d[I]}{d t}=-k_{d}[I]-\frac{[I]}{V} \frac{d V}{d t} \\
& \frac{d[S]}{d t}=-k_{s}\left[S \lambda_{0}-\frac{[S]}{V} \frac{d V}{d t}\right. \\
& \frac{d V}{d t}=-\frac{M_{0}}{\rho_{m}}\left[\varepsilon \frac{d x}{d t}+x \frac{d \varepsilon}{d t}\right] \\
& m C_{p} \frac{d T}{d t}=\left(-\Delta H_{p}\right) k_{p}[M] \lambda_{0} V-\left.U A\right|_{r}\left(T-T_{j}\right) \\
& \quad-\left.U A\right|_{\infty}\left(T-T_{\infty}\right) \\
& m_{o} C_{p_{o}} \frac{d T_{j}}{d t}=2 \alpha P+\left.U A\right|_{r}\left(T-T_{j}\right)-\left.U A\right|_{\infty \infty}\left(T_{j}-T_{\infty}\right) \\
& \lambda_{0}=\sqrt{\frac{2 f k_{d}[I]}{k_{t}}}
\end{aligned}
$$

During the free radical polymerization, three diffusion effects occur: cage, glass, and gel effects. There are different methods to model these effects. For the cage effect, the rate of initiation is simplified by incorporating primary radical termination with an initiator efficiency factor:

$$
2 f k_{d}[I]=2 k_{d}[I]-k_{t i}\left[R^{\circ}\right]^{2}
$$

CCS model (Chui, Carrat, and Soong) is used to take into consideration the gel and glass effects, Louie et al (1985). In the CCS model, diffusion is incorporated as an integrated part of the propagation and termination from the beginning until the end of the polymerization. Therefore, propagation rate constant, $k_{p}$, is variable:

$\frac{1}{k_{p}}=\frac{1}{k_{p_{0}}}+\theta_{p} \frac{\lambda_{0}}{D}$

$k_{p_{0}}$ is initial propagation rate constant. Similarly, termination rate constant, $k_{t}$, is:

$\frac{1}{k_{t}}=\frac{1}{k_{t_{0}}}+\theta_{t} \frac{\lambda_{0}}{D}$

$k_{t_{0}}$ is changing as Arrhenius function. Table 3 gives parameters of the CCS model. All the other necessary parameters and constants for this model are given somewhere else, Louie et al (1985). 
Table 3. Glass and gel effects parameters in CCS model

$\frac{k_{t}}{k_{t}^{0}}=\frac{D}{D+\theta_{p} k_{t} \lambda_{0}}, \quad \frac{k_{p}}{k_{p}^{0}}=\frac{D}{D+\theta_{p} k_{p} \lambda_{0}}$
$D=\exp \left[\frac{\left(1-\varphi_{p}\right)}{A+B\left(1-\varphi_{p}\right)}\right]$,
$A=0.168-8.21 / 10^{-6}\left(T_{c}-114\right)^{2}, \quad B=0.03$
$\theta_{p}=5.4814 \times 10^{-14} \exp (13982 / T k)$,
$\theta_{t}=\frac{2.2706 \times 10^{-23}}{[I]_{0}} \exp (17420 / T k)$
$[I]_{0}=\left[\frac{m o l}{l}\right], \quad \varphi_{p}=\frac{x(1-\varepsilon)}{1-\varepsilon x+\beta}$

Heat generation, during polymerization, should be removed by a coolant or dissipated to the environment. Otherwise, the reactor can thermally run away when the energy feeds back to increase temperature and reaction rates. Having energy balances written, polymerization can be modeled non-isothermally. The following equation shows the application of energy balance to the reactant mixture: $\frac{d T}{d t}=\frac{\left(-\Delta H_{p}\right) k_{p}[M]\left[\lambda_{0}\right] V}{m C_{p}}-\frac{U_{r} A_{r}\left(T-T_{j}\right)}{m C_{p}}$

(8)

which $m$ is the mixture mass, $C_{p}$ is the mixture specific heat, $-\Delta H_{p}$ is the heat of polymerization, $T$ is the mixture temperature, $T_{j}$ is the oil temperature, $U_{r}$ is the overall heat transfer coefficient between the reactant mixture and the jacket, and $A_{r}$ is the reactor heat transfer area. $U_{r}$ is approximated by empirical relationship [6]:

$U_{r}(x)=U_{0}[\alpha-\alpha \cdot \exp (-\beta \cdot x)]$

$A_{r}$ may be approximated by equation (2):

$A_{r}(x)=A_{0}\left(1-\frac{\varepsilon}{1+\beta} x\right)$

finally, energy balance for the reactor is:

$\frac{d T}{d t}=c_{0}\left[M \rrbracket\left[\lambda_{0}\right]-c_{1}(x)\left(T-T_{j}\right)\right.$

where

$$
\begin{aligned}
& c_{0}=\frac{\left(-\Delta H_{p}\right) k_{p} V}{m C_{p}}, \quad c_{10}=\frac{U_{0} A_{0}}{m C_{p}} \\
& c_{1}(x)=c_{10}\left(1-\frac{\varepsilon}{1+\beta} x\right)[\alpha-\alpha \cdot \exp (-\beta \cdot x)]
\end{aligned}
$$

Energy balance of oil bath is:

$$
\begin{aligned}
m_{o} C_{p_{o}} & \frac{d T_{j}}{d t}=-c_{2}(x)\left(T_{j}-T\right) \\
& -c_{3}\left(T_{j}-T_{\infty}\right)+c_{4} P-c_{5}\left(T_{j}-T_{w}\right)
\end{aligned}
$$

where $m_{o}$ is the oil mass, $C_{p o}$ is the specific heat of oil, and $T_{w}$ is the coolant water temperature. In this study, coefficients $c_{0}$ to $c_{5}$ are estimated experimentally. The results are:

$c_{10}=0.0027, \quad c_{20}=9.9584 \times 10^{-5}, \quad c_{3}=3.9811 \times 10^{-4}$, $c_{4}=5.2062 \times 10^{-5}, \quad c_{5}=6.1744 \times 10^{-4}$

States of system are defined as: $x_{1}=[M]$, $x_{2}=[I], \quad x_{3}=T, \quad x_{4}=T_{j}$, and the final state space form is:

$\frac{d x_{1}}{d t}=f_{1}\left(x_{1}, x_{2}, x_{3}\right)$

$\frac{d x_{2}}{d t}=f_{2}\left(x_{1}, x_{2}, x_{3}\right)$

$\frac{d x_{3}}{d t}=f_{3}\left(x_{1}, x_{2}, x_{3}, x_{4}\right)$

$\frac{d x_{4}}{d t}=f_{4}\left(x_{1}, x_{3}, x_{4}\right)+c_{4} u$

where $u$ is the energy input to the system:

$u=P-\frac{c_{5}}{c_{4}}\left(x_{4}-T_{\infty}\right)$

This is a non-linear model. $x=\left[[M] \quad[I] \quad T \quad T_{j}\right] \in \mathbb{R}^{4}$ is the State vector.

\section{CONTROL OF POLYMERIZATION REACTOR}

In a batch reactor, polymerization is normally carried out according to a predetermined temperature trajectory. To reach the desired properties, tight measurement and control of temperature are needed. Using appropriate temperature profile, molecular weight and its distribution can be manipulated to get desired properties of product. Non-linear control is finding new application everyday. Global Linearization Control strategy is used in this study. Initially, non-linear system is linearized in the form of input-output linearization using state feedback. Next, error is eliminated by a linear controller such as PI controller. According to this method, non-linear relation between $y$ and $u$ is transferred to a linear relation between $y$ and $v$, using suitable feedback. Assume process is described by:

$\frac{d x}{d t}=f(x)+g(x) u$

$y=h(x)$

Assume $r$ is relative order of system. $r$ is the smallest integer that satisfy $L_{g} L_{f}^{r-1} h(x) \neq 0[12] . \quad L$ is an operator defined as:

$L_{g} f(x)=\frac{\partial f}{\partial x_{1}} \cdot g_{1}+\cdots+\frac{\partial f}{\partial x_{n}} \cdot g_{n}$ 
For the present system, Relative order of system is $r=2$. if $r=n$, which $n$ order of system, system could be fully converted to the linear system. Inputoutput state linearization is:

$$
u=\psi(v, x)=\frac{v-\beta_{2} \sum_{i=1}^{4} \frac{\partial f_{3}(x)}{\partial x_{i}} f_{i}(x)-\beta_{1} f_{3}(x)-T}{\beta_{2} \cdot \alpha_{1}(x) \cdot \alpha_{4}}
$$

where $\beta_{1}$ and $\beta_{2}$ are tuning parameters. Under this feedback state, loop is defined by (Soroush and Kravaris (1992)):

$\beta_{2} \frac{d^{2} y}{d t^{2}}+\beta_{1} \frac{d y}{d t}+y=v$

A PI controller is used as the external controller:

$$
v(t)=v_{b}(t)+k_{c} e(t)+\frac{k_{c}}{\tau_{I}} \int_{0}^{\infty} e(t) d t
$$

where

$v_{b}(t)=T^{*}(t)+\beta_{1} \frac{d T^{*}(t)}{d t}+\beta_{2} \frac{d^{2} T^{*}(t)}{d t^{2}}$

Figure 2 shows the controller philosophy. Regarding dominant time constants which are $\tau_{1}=450 \mathrm{~s}$ and $\tau_{2}=50 s, \quad \beta_{1}=\tau_{1} \cdot \tau_{2} \quad$ and $\quad \beta_{2}=\tau_{1}+\tau_{2}$ are adopted. $\tau_{I}$ is selected equal to $\tau_{1}$ and $k_{c}$ is calculated based on the simulation equals 10 .

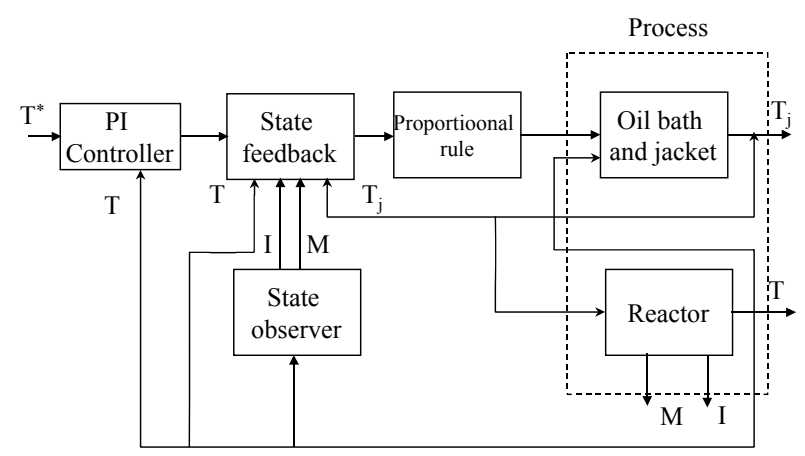

Figure 2. Structure of GLC control system

\section{RESULTS AND DISCUSSION}

Table 4 gives the experimental conditions. Polymerization initial temperature is $85^{\circ} \mathrm{C}$. Figure 3 shows the temperature trajectory, reactor temperature, as well as jacket temperature. The temperature trajectory is chosen to reduce the transfer reactions. The reactor temperature follows the temperature trajectory very well. The maximum temperature deviation is $-0.9^{\circ} \mathrm{C}$. The jacket temperature is closed to the temperature trajectory. Figure 4 shows the input $u$.
Table 4. Experimental conditions

\begin{tabular}{|l|l|}
\hline \multicolumn{2}{|c|}{ Polymerization condition } \\
\hline $600 \mathrm{ml}$ & \multicolumn{1}{c|}{ Toluene } \\
\hline $150 \mathrm{ml}$ & Methyl methacrylate \\
\hline $\begin{array}{l}\text { BPO, } 0.2 w t \% \text { of } \\
\text { monomer }\end{array}$ & Benzoyl peroxide \\
\hline $150 \mathrm{rpm}$ & Mixing speed \\
\hline
\end{tabular}

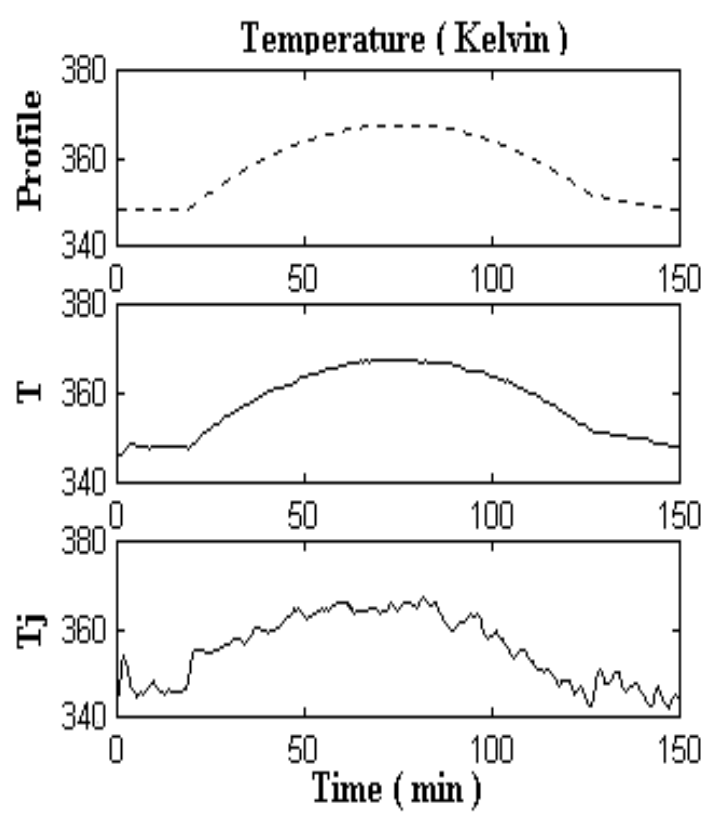

Figure 3. The temperature trajectory, reactor and jacket temperatures

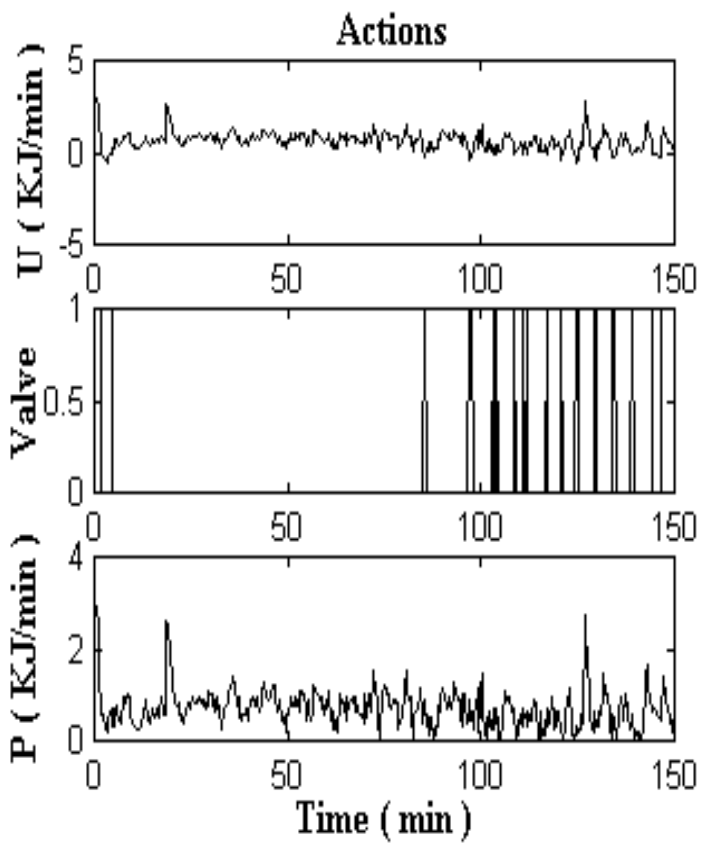

Figure 4. Input of the system 
Controller performance is very good. Therefore, it is expectable that other classic method for controller design could be successful.

\section{CONCLUSION}

A Globally Linearizing Control (GLC) strategy is applied to control the temperature of a batch reactor. In this paper, an oil bath is constructed for the heating and cooling purposes. Then, a mechanistic model is developed for the solution polymerization of methyl methacrylate in a batch reactor. An external linear PI controller is coupled to eliminate the error. The controller performance is very good and the previously developed GLC method is suitable for the oil heating system.

\section{ACKNOWLEDMENT}

The author acknowledges the financial support of AmirKabir University of Technology, also valuable help of Dr. Falah and Mr. Maleki in electronic circuits.

\section{REFERENCES}

Berber R., K. Yetik, and A. Cahmh (1998). Nonlinear Model Predictive Control with State Estimation in Batch Polymerization. American Control Conference, 3778-3782.

Chylla, R. W., D. R. Haase (1990). Temperature Control of Semibatch Polymerization Reactors. AICHE National Meeting, Chicago.

Clarke-Pringle T., and J. F. MacGregor (1997). Nonlinear Adaptive Temperature Control of Multi-Product, Semi-Batch Polymerization Reactors. Comp. Chem. Engng., 21, 1395-1409.

Louie, B. M., Carratt, G. C., and Soong, D. S. (1985). Modeling the Free Radical Solution and Bulk Polymerization of Methyl Methacrylate. J. Appl. Polym. Sci., 30, 3985-4012.

Mendoza-Bustos S. A., Penlidis A., and Cluett W. R. (1990) Use of a Modern Polymerization Pilot-Plant for Undergraduate Control Projects. Chem. Eng. Education, Winter, 34-39.

Mutha R. K., W. R. Cluett, and A. Penlidis (1997). On-Line Nonlinear Model-Based Estimation and Control of a Polymer Reactor. AIChE $J ., 43,3042-3058$.
Penlidis A., S. R. Ponnuswamy, C. Kiparissides and K. F. O’Driscoll (1992) Polymer Reaction Engineering: Modeling Consideration for Control Studies. The Chem. Eng. J., 50, 95-107.

Peterson T., E. Hernandez, Y.Arkun and F. J. Schork (1989) Nonlinear Predictive Control of a Semibatch Polymerization Reactor by an Extended DMC. American Control Conference, 1534-1539.

Slotine, E., W. Li (1991) Applied Nonlinear Control. Prentice Hall Co., New York.

Soroush M. and C. Kravaris (1992) Non-linear Control of a Batch Polymerization Reactor: An Experimental Study. AIChE J., 38, 1429-1448.

Tzouanas V. K., Shah S. L. (1989) Adaptive PoleAssignment Control of a Batch Polymerization Reactor. Chem. Eng. Sci., 44, 1183-1193.

Wright R. A., and C. Kravaris (1997) Practical Development of a Nonlinear Controller for an Industrial Scale Batch Polymerization Reactor. American Control Conference, 724-729. 